\title{
Incidence and Complication Rates for Total Hip Arthroplasty in Rheumatoid Arthritis: A Systematic Review and Meta-Analysis Across Four Decades
}

\author{
Owen Taylor-Williams (D) · Johannes Nossent · Charles A. Inderjeeth
}

Received: August 20, 2020 / Accepted: September 19, 2020 / Published online: September 30, 2020

(C) The Author(s) 2020

\begin{abstract}
Introduction: Over the past several decades, management of rheumatoid arthritis (RA) has evolved significantly, but few studies have examined the real-world impact of these changes on orthopaedic surgery in patients with RA. This systematic review assessed total hip arthroplasty (THA) incidence and postoperative complication rates across the past four decades. Methods: This is a systematic literature review sourcing data on THA in patients with RA from the electronic databases MEDLINE, EMBASE, Scopus, and Cochrane between January 1, 1980 and December 31, 2019.
\end{abstract}

Digital Features To view digital features for this article go to https://doi.org/10.6084/m9.figshare.12967229.

Electronic Supplementary Material The online version of this article (https://doi.org/10.1007/s40744$020-00238-z)$ contains supplementary material, which is available to authorized users.

O. Taylor-Williams $\cdot$ J. Nossent $(\square)$.

C. A. Inderjeeth $(\bowtie)$

School of Medicine, The University of Western

Australia, Perth, Australia

e-mail: johannes.nossent@health.wa.gov.au

C. A. Inderjeeth

e-mail: Charles.Inderjeeth@health.wa.gov.au

J. Nossent . C. A. Inderjeeth

Sir Charles Gairdner and Osborne Park Health Care

Group, Perth, Australia
Results: The search retrieved 1715 articles of which 44 were included for quantitative synthesis. The rate for THA decreased by almost $40 \%$ from $11 / 1000$ patient years (PY) in the 2000 s to $7 / 1000 \mathrm{PY}$ in the 2010 s, while the overall complication rate decreased from 9.9\% in the 1990 s to $5.3 \%$ in the 2010 s. Throughout the duration of the study, THA incidence and overall complication rate decreased. However, not all individual complication rates decreased. For example, revision and periprosthetic fracture decreased, infection and aseptic loosening remained constant, and dislocation increased.

Conclusion: Medical management of patients with RA has reduced the need for THA, while postoperative medical and surgical management has improved some postoperative outcomes. Nevertheless, there remains room for further improvement to postoperative outcomes through RA-specific management.

Keywords: Complications; Disease-modifying antirheumatic drugs; Incidence; Rheumatoid arthritis; Systematic review; Total hip arthroplasty 


\section{Key Summary Points}

This systematic review analysed published literature on total hip arthroplasty (THA) in patients with rheumatoid arthritis (RA) to estimate the incidence of THA and postoperative outcomes (revision, periprosthetic fracture, infection, aseptic loosening, dislocation, venous thromboembolism (VTE), and mortality) in patients with RA throughout the past four decades.

Medical management has resulted in a decreasing incidence of THA in patients with RA, while surgical and medical management has improved postoperative outcomes for patients with RA.

Not every postoperative outcome has improved: revision and periprosthetic fracture rates have decreased, infection and aseptic loosening have remained constant, and dislocation has increased.

Specific changes to RA management which have reduced THA incidence are likely to include acceptance of methotrexate (MTX) as first-line therapy, earlier initiation of synthetic diseasemodifying antirheumatic drugs (sDMARD) therapy, and the introduction of biological disease-modifying antirheumatic drugs (bDMARDs).

Factors involved in changing complication rates include changing demographics, use of sDMARD and bDMARD therapy, newer surgical approaches, and improved thrombophylaxis.

\section{DIGITAL FEATURES}

This article is published with digital features to facilitate understanding of the article. To view digital features for this article go to https://doi. org/10.6084/m9.figshare.12967229.

\section{INTRODUCTION}

Over the past several decades, medical management for rheumatoid arthritis (RA) has evolved significantly with the increased use of early, targeted therapies to take advantage of the 'window of opportunity', and the addition of newer synthetic disease-modifying antirheumatic drugs (sDMARDs) aiming for a low state of disease activity or remission [1-3]. Despite pharmacological therapy, disease progression may reach a stage where patients require surgery for joint damage [1]. Surgery, being at the end of the treatment protocol $[1,4]$, is a good indicator for the summative effect of all interventions used to treat RA [1, 5]. Consequentially, measuring operation rates will provide an improved understanding of how changes to RA management have impacted patient outcomes and resource allocation [6, 7]. Rates of total hip arthroplasty (THA) have increased in the general population [8], but a decline has been reported for patients with RA which appears to have started before the introduction of biological DMARD (bDMARD) [9-11]. This paper will investigate THA incidence in patients with RA over time to help determine whether changes in RA management may have affected operation rates.

Uncemented THA procedures introduced in the late 1970s to reduce complication rates, such as osteolysis, are currently preferred by many surgeons [12]. Research into orthopaedic surgery outcomes in patients with RA is scarce. However, an increased risk of complications for patients with RA, compared to the general population, has been postulated due to the complex pathogenesis of RA, use of immunomodulating drugs, and increased prevalence of joint deformity [13]. Some, but not all studies, report a higher frequency of dislocation, infection, venous thromboembolism (VTE), and periprosthetic fracture after total joint replacement (TJR) in patients with RA compared to patients with osteoarthrosis (OA) [14-19]. A possible reason for the reported 
discrepancies is that studies were inadequately powered [19], something which could be better assessed by a systematic review and meta-analysis. Knowing THA complication rates (risk-benefit) is essential to patients and surgeons when considering surgery.

This paper aims to determine overall and temporal rates of THA in patients with RA across the past four decades and the overall and temporal rates of complications including specific rates for VTE, infection, dislocation, periprosthetic fracture, aseptic loosening, revision, and mortality.

\section{METHODS}

\section{Study Design}

The guidelines for systematic reviews of prevalence studies by the Joanna Briggs Institute (JBI) [20] and the Preferred Reporting Items for Systematic Reviews and Meta-Analyses (PRISMA) 2009 recommendations were followed [21]. Medical Subject Headings, keywords, and Boolean operators were used when searching the electronic databases MEDLINE, EMBASE,
Scopus, and Cochrane. No separate searches for grey literature were done. The search strategy was peer-reviewed between the first author, chief investigator, and senior librarian (see Table 1). Search results were loaded into EndNote X8 (Clarivate Analytics, PA, USA) and duplicates removed. Next, titles and abstracts of studies were screened and included for further review if articles were in English and reported on primary or secondary outcomes of interest. If it was uncertain whether a study met inclusion or exclusion criteria, this was discussed with the chief investigator. Then, included studies were reviewed in full, and excluded if the exclusion criteria were met. Following this, the studies' reference lists were searched for further relevant studies.

This article is based on previously conducted studies and does not contain any studies with human participants or animals performed by any of the authors.

\section{Inclusion Criteria}

Studies providing sufficient data to calculate the primary outcomes, rates of primary THA or THA complications in patients with RA, were

Table 1 Abbreviated search strategy with concepts for the search in the header rows, and search terms for each concept listed below

\begin{tabular}{|c|c|c|c|c|c|c|}
\hline Rheumatoid arthritis & AND & Total hip replacement & AND & Rates & AND & Adult \\
\hline Rheumatoid arthritis & & Total hip replacements & & Rate(s) & & Adult \\
\hline OR & & OR & & OR & & OR \\
\hline \multirow[t]{7}{*}{ Rheumatic disease } & & Total hip arthroplasty/total hip arthroplasties & & Incidence & & Aged \\
\hline & & OR & & OR & & OR \\
\hline & & Total hip implantation(s) & & Prevalence & & Middle- \\
\hline & & OR & & OR & & aged \\
\hline & & Hip prosthesis implantation & & Trend(s) & & \\
\hline & & & & OR & & \\
\hline & & & & Epidemiology & & \\
\hline
\end{tabular}

Each column is representative of the parenthesis used in the search, with terms combined using Boolean operators (AND and OR) as shown. For the full search strategy (how it was entered into the MEDLINE database), please see the supplementary material 
included on the basis of the remainder of the inclusion criteria (see "Data Synthesis and Analysis" section for more detail). Similar criteria applied for the secondary outcomes, dislocation, VTE, infection, aseptic loosening, 90-day mortality, postoperative periprosthetic fracture, and revision rates. Rates were taken as averages, across each decade and continent. Studies included for quantitative analysis also had to be of a cohort, cross-sectional, or randomised control trial design and published from January 1, 1980 to December 31, 2019.

\section{Exclusion Criteria}

Studies were excluded if patients did not have adult-onset RA and were (a) non-research papers (editorials, letters, conference proceedings/abstracts); (b) systematic reviews; (c) animal models; (d) case report or case series. For studies with overlapping populations, reporting the same outcomes, the larger study was included and the smaller study excluded; but if different outcomes were reported, both were included.

\section{Data Abstraction}

In addition to primary and secondary outcomes, information on the general study characteristics, population, and methodology was collected. General study characteristics extracted included (a) author; (b) year of publication; (c) location where the study was conducted. Population data that was extracted include (a) sample size; (b) median age; (c) number of male and female subjects; (d) body mass index. Methodological information that was collected included (a) sampling method; (b) definition of RA (versus secondary $\mathrm{OA}$ ); (c) follow-up duration.

\section{Data Synthesis and Analysis}

Studies were initially assessed using Hoy's et al. risk of bias tool, before inclusion in the review [22]. Since the median number of studies in a meta-analysis on the Cochrane Database for Systematic Reviews is three, a meta-analysis on pooled results was carried out if there were three or more studies [23]. Individual complication rates were calculated by dividing the number of patients with RA with complications after THA by the total number of patients with RA undergoing THA. The overall complication rate was calculated by adding together the rates of dislocation, VTE, infection, aseptic loosening, and revision for each study. THA rates were calculated by dividing the number of patients with RA receiving THA by the total number of RA patient years (PY). If PY were not provided, they were calculated by multiplying the mean number of follow-up years by the number of patients. If a mean was not published, then the median was used as a substitute for the mean. For THA incidence, studies were pooled, analysed, and compared by decade of publication, continent of origin, and whether an attempt was made to exclude patients with OA. Postsurgical outcomes were analysed by the decade of publication, continent of origin, and surgical outcomes reported (dislocation, VTE, infection, aseptic loosening, mortality, and revision). A random effect model with a Freeman Turkey Transformation on the System for the Unified Management, Assessment and Review of Information (JBI, Adelaide, SA) was used for analysis. Study heterogeneity was tested using the $I^{2}$ statistic and represented visually using forest plots.

\section{RESULTS}

The electronic search carried out on February 6, 2020 produced 1715 results. Removal of duplicates eliminated 823 results, leaving 892 articles' titles and abstracts to be reviewed. Of these 892 articles, 140 were reviewed in full and resulted in 38 articles being included. References for the 38 included articles were then searched using the same inclusion criteria as the electronic search, resulting in an additional six articles being included. From these 44 articles, 12 were included exclusively in the incidence analyses [11, 24-34], 31 exclusively in the complications analyses [16, 35-64], and one article in both sections [65] (see Fig. 1). 


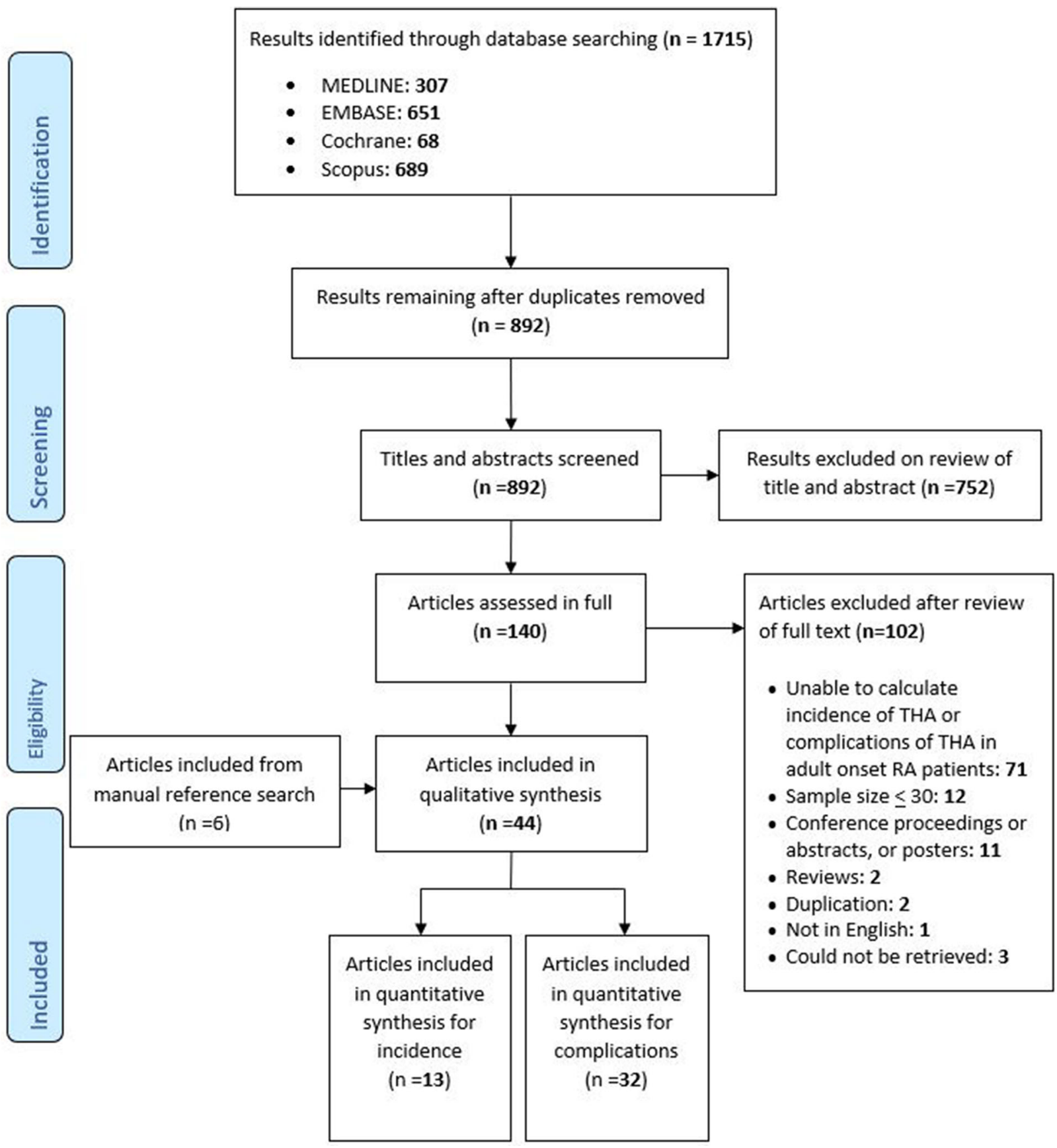

Fig. 1 PRISMA flow chart

\section{Included Studies}

\section{Incidence}

\section{Distribution of Studies}

The 13 studies analysed for THA incidence originated from 11 countries [11, 24-34, 65], mostly in Europe, with the remainder from North America and Asia. Temporal distribution was weighted towards the later decades, with the most studies published from 2010 to 2019. Unfortunately, as a result of no studies being published in the 1980s, and only one study in the 1990s, analysis of temporal trends could not 
be analysed pre 2000s. In all 617,096 PY, from patients with diagnosed RA and no previous THA, were included from studies ranging in size from $452 \mathrm{PY}$ to $161,129 \mathrm{PY}$, with a mean of $47,468 \mathrm{PY}$ and standard deviation of 47,468 PY.

\section{Exclusion and Inclusion Criteria of Included Studies}

Two articles specifically excluded patients with OA [33, 65], while 11 articles did not $[11,24-32,34]$. No articles reported the clinical indications for THA such as pain, functional limitations, or stiffness [66]. No papers used comorbidities as exclusion criteria.

\section{Risk of Bias and Study Type}

All studies included were cohort studies and deemed as having a low risk of bias $[11,24-34,65]$. Six articles did not verify the RA diagnosis via the American College of Rheumatology (ACR) 1987 or American Rheumatology Association (ARA) 1956 criteria $[11,25-28,30]$. Two articles had a loss to followup exceeding $20 \%$ and lacked analysis as to whether this resulted in a non-response bias $[31,34]$.

\section{Complications}

\section{Distribution of Studies}

Thirty-two articles reporting on the complications of infection (22 articles) [16, 35-41, $43,44,47,48,51,52,55-60,62,64]$, overall revision (21 articles) [16, 35, 36, 38, 39, 41, $42,44,50-55,57-59,62-65]$, aseptic loosening (15 articles) $[35,36,38,39,42,46,50-52$, $55,57-59,61,63]$, dislocation (13 articles) $[16,35,36,38,39,41,42,47,49,50,52,59,63]$, mortality (5 articles) [16, 45, 48, 60, 64], periprosthetic fracture (11 articles) $[16,36,38$, $39,41,44,47,50,58,59,63]$, and VTE (5 articles) $[16,52,62-64]$ were analysed. Studies came from four continents and 15 countries. By continent, Europe and North America published the most articles, and by country the USA published the most papers followed by Japan. With only two articles from 1980 to 1989 , the temporal analysis of articles was commenced from 1990 and was approximately even from 1990 to 2019.

\section{Risk of Bias}

Risk of bias was deemed as low for 30 articles $[16,35-60,63-65]$, and moderate for two articles [61, 62]. Of these 35 articles, 22 did not use the ARA 1956 or ACR 1987 criteria [16, 36, $38,41-54,57-61]$, and 20 did not provide adequate definitions of complications [16, 36, 38, $41-43,46-53,56,59-62,65]$. As a result, it was deemed that 25 articles did not provide adequate definitions $[16,36,38,40-43,45-54$, $56-62,65]$. From the 35 articles, seven had potentially biased cohorts [56-58, 60-62, 65], and four had a risk of non-response bias $[16,39,55,59]$. Three studies were potentially biased in participant selection $[55,59,61]$, and two did not gather information directly from patients or their medical records $[54,62]$

\section{Findings}

Results are presented in the text if three or more studies were available for quantitative analysis, as it was deemed that the analysis of fewer than three studies might be unrepresentative. For completeness, the full set of results, including where the threshold of three studies was not achieved, is illustrated in Table 2 and 3. Additionally, for the full set of forest plots, see the supplementary file.

\section{THA Incidence Rate in RA}

THA incidence decreased by almost $40 \%$ from $11 / 1000$ with a $95 \%$ confidence interval (CI) of $7-16 / 1000$ in the 2000 s, to $7 / 1000$ PY (CI 6-9) in the 2010s, resulting in a THA incidence rate of 7/1000 PY with CI 6-9/1000 (Fig. 2) across the full observation period (1990-2019). By continent, Europe had an incidence rate of 8/1000 PY (CI 6-10 PY), while estimates of incidence in North America (6/1000 PY; CI 2-13) and Asia (6/1000 PY; CI 6-7) were based on a limited number of studies. See Table 2. 
Table 2 Summary of the incidence of THA in patients with RA analysed by decade, continent, and diagnosis

\begin{tabular}{ll}
\hline Category & $\begin{array}{l}\text { Incidence per 1000 PYs } \\
(\text { CI })\end{array}$ \\
\hline Overall rate 1980-2019 & $7(6-9)$ \\
By decade & \\
$1990-1999$ & $46(29-68)^{\mathrm{a}}$ \\
$2000-2009$ & $11(7-16)$ \\
$2010-2019$ & $7(6-9)$ \\
By continent & $8(6-10)$ \\
Europe & $6(2-13)^{\mathrm{a}}$ \\
North America & $6(6-7)^{\mathrm{a}}$ \\
Asia & \\
By diagnosis & $7(3-12)^{\mathrm{a}}$ \\
OA specifically excluded & $7(5-9)$ \\
OA not specifically \\
excluded
\end{tabular}

${ }^{a}$ Not enough studies to meet the three studies required for quantitative analysis, and rate should be used and interpreted with caution as it may not be representative of the broader population

\section{THA Complication Rates in RA (Overall)}

Complication rates for the full observation period (1980-2019) averaged $12.8 \% \quad$ (CI 8.4-17.9\%), but over time increased from 9.9\% (CI $6.2-14.4 \%$ ) in $1990-1999$ to $12.7 \%$ (CI 7.3-19.2\%) in 2000-2009, and decreased to $5.3 \%$ (CI 2.5-8.9\%) in 2010-2019 (Fig. 3). Complication rates were highest in Europe (9.9\% CI 6.6-13.7\%), followed by North America $(7.2 \%$ CI $3.4-12.3 \%)$ and Asia $(4.5 \%$ CI $1.9-8.0 \%)$. Again, there was significant heterogeneity despite subgroup analysis by complication (revision, VTE, dislocation, aseptic loosening, and infection) and decade (1980-1989, 1990-1999, 2000-2009, 2010-2019).

\section{THA Complication Rates by Individual Complications}

The overall rate of revision from 1980 to 2019 was $8.8 \%$ (CI $6.1-11.9 \%$ ) but decreased from 8.1\% (CI 4.4-12.8\%) and 8.5\% (CI 4.7-13.2\%) in the $1990 \mathrm{~s}$ and $2000 \mathrm{~s}$, respectively, to $6.2 \%$ (CI $2.3-11.5 \%$ ) in the 2010s. Similarly, periprosthetic fracture rates decreased from $1.0 \%$ (CI $0-3.9 \%$ ) in the 2000 s to $0.4 \%$ (CI

Table 3 Summary of the estimated postoperative complication rates for patients with RA receiving THA

\begin{tabular}{lllllc}
\hline & \multicolumn{5}{l}{ Percentage of complications $(\mathbf{C I})$} \\
\cline { 2 - 6 } & $\mathbf{1 9 8 0 - 1 9 8 9}$ & $\mathbf{1 9 9 0 - 1 9 9 9}$ & $\mathbf{2 0 0 0 - 2 0 0 9}$ & $\mathbf{2 0 1 0 - 2 0 1 9}$ & $\mathbf{1 9 8 0 - 2 0 1 9}$ \\
\hline VTE & $2(0-6.1)^{\mathrm{a}}$ & No studies & $1.9(0-7.9)^{\mathrm{a}}$ & $0.3(0-0.6)$ & $0.4(0-1.2)$ \\
Dislocation & No papers $^{\mathrm{b}}$ & $1.1(0-4.3)^{\mathrm{a}}$ & $0.4(0.1-0.8)$ & $1.5(0.5-3)$ & $0.8(0.2-1.7)$ \\
Periprosthetic fracture & No papers $^{\mathrm{b}}$ & $0.6(0.2-1.1)^{\mathrm{a}}$ & $1.0(0-3.9)$ & $0.4(0.1-0.8)$ & $0.3(0.1-0.6)$ \\
Aseptic loosening & $16.2(5.8-30.1)^{\mathrm{a}}$ & $2.8(1.5-4.5)$ & $3.8(1.4-7.2)$ & $3.8(0-11.9)$ & $4.0(2.2-6.4)$ \\
Infection & $2.7(0.3-6.7)^{\mathrm{a}}$ & $2.3(1.1-3.9)$ & $2.3(0-8.7)$ & $2.6(0.8-5.2)$ & $2.6(1.4-4.2)$ \\
Revision & $12.9(0-42.3)^{\mathrm{a}}$ & $8.1(4.4-12.8)$ & $8.5(4.7-13.2)^{\mathrm{a}}$ & $6.2(2.3-11.5)$ & $8.8(6.1-11.9)$ \\
90-day mortality & No papers & $0^{\mathrm{a}}$ & $0.7(0.3-1.4)^{\mathrm{a}}$ & $0.6(0.2-1.1)$ & $0.6(0.2-1.1)$ \\
Complication rate & $25.1(0-70.8)^{\mathrm{a}}$ & $9.9(6.2-14.4)$ & $12.7(7.3-19.2)$ & $5.3(2.5-8.9)$ & $12.8(8.4-17.9)$ \\
\hline
\end{tabular}

${ }^{a}$ Insufficient number of studies to meet the cut-off criteria of three studies, and hence these results should be used and interpreted with caution as they may not be representative of the broader population

b Category could not be calculated as a result of limited studies 


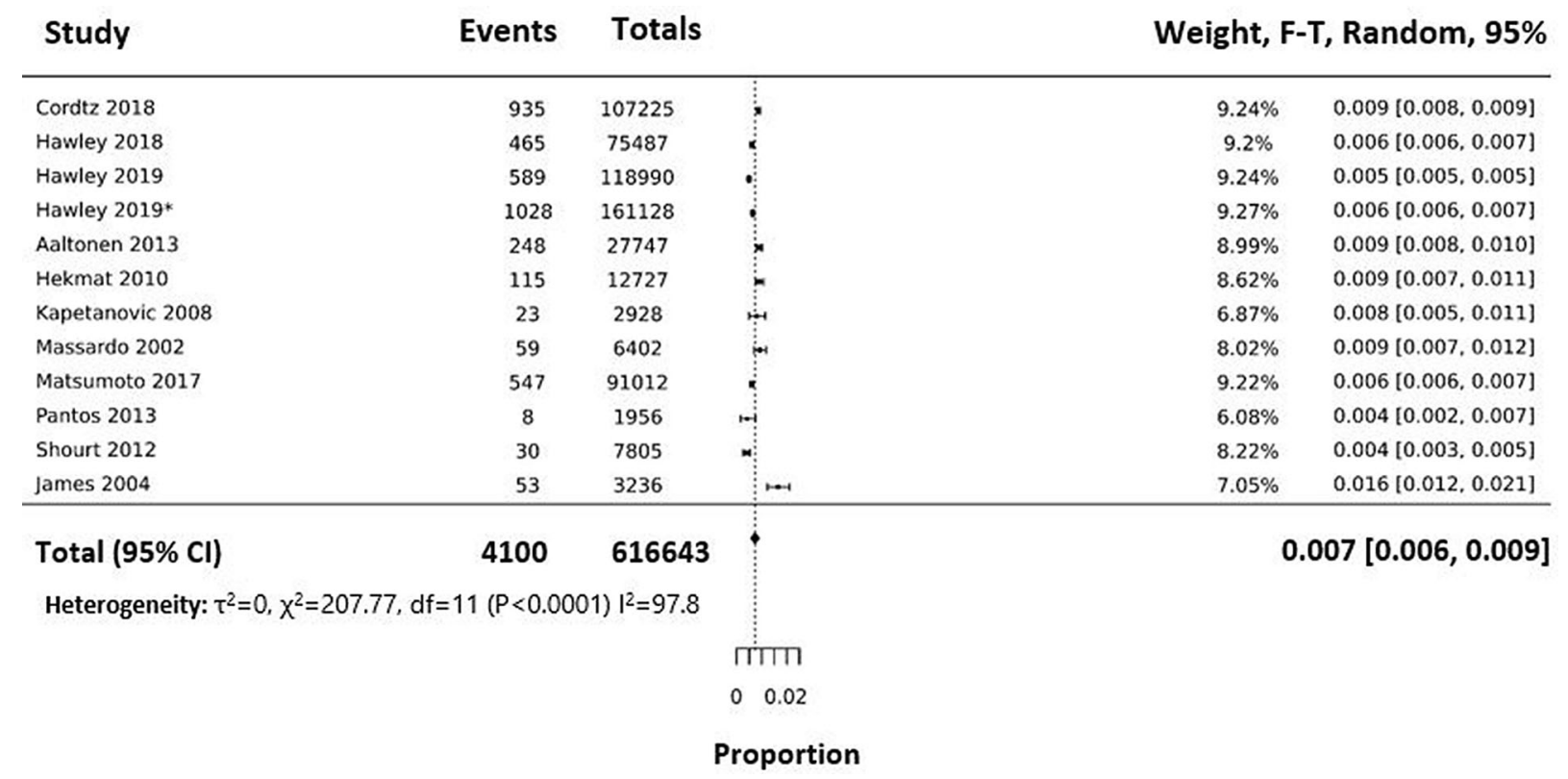

Fig. 2 Forest plot representing the distribution of the studies analysed for the incidence of THA in patients with RA. Events are the numbers of THA occurring in patients with RA in each study, while the total is the number of PY observed. The dotted line represents the mean effect,

0.1-0.8) in the 2010s, resulting in an overall rate from 1990 to 2019 of $0.3 \%$ (CI $0.1-0.6 \%$ ).

Infection rate from 1980 to 2019 was $2.6 \%$ (CI 1.4-4.2\%) with similar rates observed in all three decades. Aseptic loosening rates from 1980 to 2019 were $4.0 \%$ (CI 2.2-6.4\%), but increased from $2.8 \%$ (CI 1.5-4.5\%) in the $1990 \mathrm{~s}$ to $3.8 \%$ (CI $1.4-7.2$ in 2000 s and CI $0-11.9$ in 2010s) in the subsequent decades.

The dislocation rate for 1990-2019 was 0.8\% (CI $0.2-1.7 \%$ ) but increased from $0.4 \%$ (CI $0.1-0.8 \%$ ) in the 2000 s to $1.5 \%$ (CI $0.5-3.0 \%$ ) in the 2010s.

VTE and 90-day mortality had insufficient data to determine a temporal trend. Overall rates of suspected VTE and mortality were $0.4 \%$ (CI $0-1.2 \%$ ) and $0.6 \%$ (CI $0.2-1.1 \%$ ), respectively.

These results are summarised in Table 3.

\section{DISCUSSION}

This meta-analysis demonstrates that THA incidence and postoperative complications rates have where mean and confidence intervals of each of study are represented by the box and whiskers. The total effect is represented by the diamond at the bottom. Rates in the right-hand column are quoted in THA per single PY

decreased over the last 30 years. The change in complication rates results from the rates of revision and periprosthetic fracture decreasing, infections and aseptic loosening remaining stable, and dislocation increasing. As for mortality and VTE, there was insufficient data to determine a trend.

\section{Incidence}

\section{Temporal Variations}

Decreasing rates of THA in patients with RA are consistent with numerous other studies [67-69] with a current incidence rate of 7/1000 PY. Interestingly, this differs to the findings from the UK Early RA Study and UK Early RA Network, which suggest that there has been no significant change for THA rates in patients with RA. This difference may be because the study by Nikiphorou et al. (2014) was not large enough (did not have adequate power), was UK specific, or did not exclude secondary THA (excluded in our review) [70]. It has previously been identified that worse disease activity is associated with poorer functioning and 


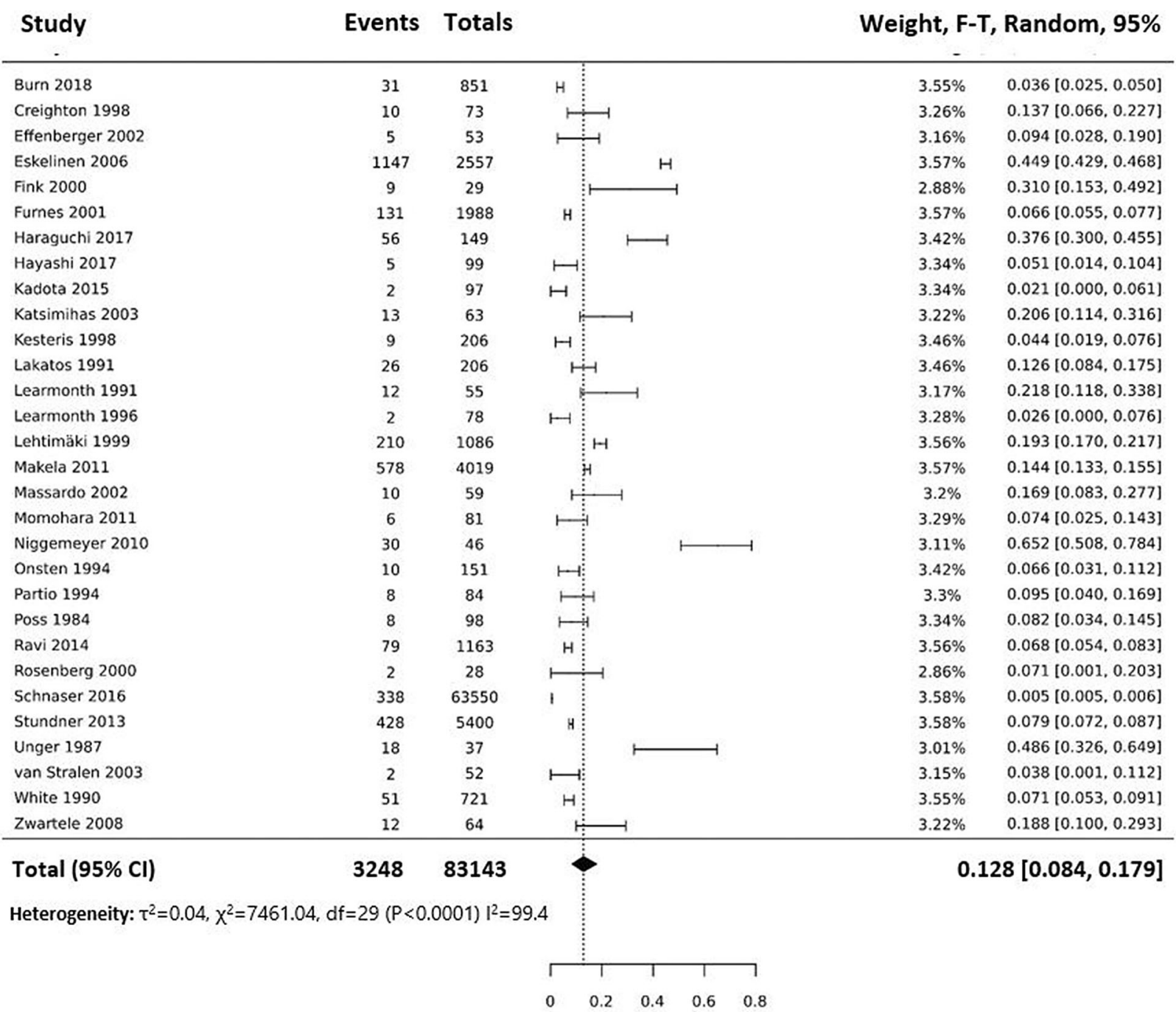

Proportion

Fig. 3 Forest plot representing the distribution of the studies analysed for postoperative complications of THA in patients with RA. Events are the total numbers of VTE, dislocation, aseptic loosening, revision, infection, and dislocation reported for each study, while the total is the

increased incidence of orthopaedic surgery [5]. Hence, while there are multiple potential reasons for decreasing THA incidence, a major contributing factor may be the more effective management protocols and treatments that focus on minimising RA disease activity $[3,71,72]$. Specific changes to management protocol include the widespread acceptance of MTX as first-line in the 1990s [3, 71, 73], earlier initiation of sDMARDs from the late 1980s to number of patients observed for each study. The dotted line represents the mean effect, where the mean and confidence intervals of each study are represented as box and whiskers plots. The total effect is represented by the diamond at the bottom

the mid 2010s [3, 71, 74-76], and introduction of bDMARDs in the early to mid 2000s $[3,72,77,78]$. Since the time between RA diagnosis and THA is approximately 15 years [79], the almost 40\% decrease decreasing incidence of THA from the 2000s to 2010s is likely the result of changes that occurred in the 2000-2009 decade. While earlier initiation of sDMARD therapy now results in approximately half of patients with incident RA receiving 
sDMARD therapy in the first year [74-76], it is difficult to quantify the significance of this earlier initiation in THA incidence. Another potential contributing factor to the decreasing incidence of THA is a less severe natural course of RA $[80,81]$. However, this would be expected to result in a gradual change in THA incidence and not the nearly $40 \%$ decrease that was observed in the study. In summary, while it is difficult to select any single one of these scenarios, on the basis off the timeline of change and the almost $40 \%$ decrease in the incidence of THA between the 2000s and 2010s, there would appear to be considerable support for the significance of early MTX and bDMARD therapy in the prevention of THA.

\section{Variation in THA by Continent}

The THA rate was higher in Europe than North America, but with overlapping confidence intervals the significance is uncertain. Considering that the reported prevalence of RA is higher in North America [82, 83], it seems unlikely that the higher rate of THA in European patients with RA can be explained on the basis of RA characteristics alone. With THA also being more prevalent in the general European population [84], this suggests that a non-RAspecific factor is involved in this intercontinental variation of THA rates. A probable cause is due to the difference in health care systems between Europe and North America (universal health care versus private health coverage), as cost and access to health care are known to influence the rate of TJR $[85,86]$.

\section{Complications}

\section{Overall Complication Rates}

The combined complication rate increased from the 1990s to 2000s and then decreased to the 2010s. In addition to the factors influencing individual complications discussed in the next section, more systemic causes, such as changes to training, could also have influenced the changes in complication rates. The $12.8 \%$ overall complication rate is greater than the $7 \%$ complication rate in the GLORY study, which looked at a similar set of complications [87]. Since the GLORY study was carried out in the general population receiving THA [87], the higher rate of complications found in this study would go on to suggest at an increased risk of complications in patients with RA following THA and that RA-specific management could help to reduce this complication rate.

Higher rates of THA complications in Europe followed by North America and Asia may seem contradictory to the earlier finding that THA rates are more common in Europe and North America, because of the previously suggested correlation between lower complication rates and higher operation volumes [88, 89]. However, incidence rates are a reflection of the proportion of patients requiring THA, and not the absolute operative volume. Additionally, if THA is interpreted as an indicator of disease severity $[1,4]$, and complications are a potential result of disease severity [90, 91, 92-94], this would explain the higher rates of complications in the places with higher incidence rates. An alternative explanation for the variation in complication rates might be that the demographics of patients considered for surgery differs between each of these continents.

\section{Complication Rates by Individual Complications}

THA revision rates appear to have remained constant from 1990 to 2009 and then decreased for the 2010-2019 period. Since this trend was not mirrored in the general population [95-97], it suggests that the causative factors of the trend were RA specific and not the result of general changes to THA pre- and postoperative management. The most common causes of THA revision in the general population are dislocation, loosening, and infection [96]. Nevertheless, as will be discussed, dislocation rates increased, while loosening and infection rates remained constant, which would suggest that revision rates should increase rather than decrease. One potential explanation for this is that clinicians are favouring less invasive methods of correction than full revisions. For example, in the case of infections, clinicians 
may be managing these more through more potent bone- and joint-specific antibiotics as opposed to surgical interventions in earlier years. Another potential explanation is that the wide confidence intervals due to a limited number of studies have obscured the actual trend in each of these subcategories. However, a further explanation is that the causes of revision other than dislocation, loosening, and infection have been the cause of decreasing revision rates.

Dislocation rates increased from the 2000s to the 2010s. Potential explanations for this increase include age, femoral head size, prosthetic development, and surgical approach/ technique [97]. Of these potential factors, surgical approaches with lower dislocation rates have been gaining popularity [98]; and femoral head size has been increasing, which reduces rather than increase dislocation risk [99]. Age then seems a likely explanation as it has been documented that patients receiving THA are becoming younger and living longer, providing more time for a dislocation to occur [100].

Aseptic loosening rates appear to have remained constant from 1990 to 2019 in THA in patients with RA but decreased in the general population [101, 102]. This was likely the result of few aseptic loosening events being recorded, hence resulting in wide confidence intervals masking any changes. Since disease activity is a risk factor for aseptic loosening [92], then worsened RA control could be a potential explanation for the increased rates, but this would be contradictory to the other findings in this review, and thus it was deemed unlikely.

Rates of infection remained unchanged since the 1990s, at approximately $2.6 \%$, which would appear to be two to six times that in the general population [103]. One likely reason for this elevated risk is the use of corticosteroids and their impacts on immune suppression [104, 105]. Interestingly, infection rates have remained unchanged, while sDMARD, bDMARD, and corticosteroid use has increased. But, mean corticosteroid dose has remained constant (mean 4.9-5.8 mg) [3, 72, 77, 78, 106]. This appears to contradict numerous studies which suggest that patients with RA receiving bDMARDs are at a higher risk of infection than those receiving sDMARDs [107-110]. It is also worth noting that there is no difference in infection risk between the various bDMARDs [111], and hence development of new bDMARDs has not impacted infection risk. Given the rates are unchanged, this would suggest that other changes in management, such as improved infection prophylaxis, or sDMARD and bDMARD breaks perioperatively, have compensated for the increased infection risk. Alternatively, this would lend support to the studies which suggest that patients on sDMARDs and bDMARDs are not at an elevated risk of infection [104, 105].

Since 2000, periprosthetic fracture rates appear to have decreased in patients with RA, but increased in the general population, resulting in a lower fracture rate in patients with RA than the general population $(0.3 \%$ versus $0.9 \%)$ [94]. Reasons for the increasing rates in the general population include a demographic shift, whereby older and younger patients are operated on, and increasing life expectancy, resulting in more follow-up time for complications to occur [112]. The opposing trend for THA in patients with RA suggests the role of a change specific to patients with RA. One RA-specific change is the introduction of bDMARDs, which through decreased disease activity results in a slower decline of bone mineral density (BMD) [90, 91, 93, 94]. However, the periprosthetic fracture risk could also have been influenced by increased sDMARDs availability or caution when prescribing corticosteroids.

The $0.4 \%$ rate of VTE in patients with RA post THA is lower than the $0.7-1.6 \%$ rate of VTE found in the general population after THA $[87,113]$. While this would suggest that patients with RA are at a decreased risk of VTE post surgery, there was a wide confidence interval. However, the VTE rate in patients with RA after THA does not exceed that of the OA and general population, as suggested by Ravi et al. [19]. Within the general population thrombophylaxis and minimally invasive surgical techniques have been identified as significant factors in achieving the current low rate of VTE [113-116] and our data indicate that this extends to patients with RA as well.

The 90-day mortality rate of $0.6 \%$ after THA in patients with $\mathrm{RA}$ is greater than the 
$0.1-0.24 \%$ mortality rate found in the general population [117, 118]. This differs from other studies where no difference in the rates of inhospital mortality was observed following total knee arthroplasty (TKA) in patients with RA or OA [119]. This could either suggest that patients with RA have an increased risk of mortality in the time after leaving the hospital or that the relative risk of patients with RA versus patients with OA undergoing THA is greater than that of patients with RA versus patients with $\mathrm{OA}$ undergoing TKA.

\section{STRENGTHS AND LIMITATIONS}

At the time of writing, this is the first systematic review to estimate the rate of THA in patients with RA and the risk of complications, providing valuable information to patients, clinicians, and researchers. The use of multiple, diverse outcomes provides a broad and comprehensive overview of the state of surgical and medical management of patients with RA at a population level.

Throughout this report, emphasis has been placed on presenting clinically significant information. Specifically, complication rates have been used as outcomes as they are of clinical significance, but they are also a raw form of data significantly dependent on follow-up time. This can result in an apparent inconsistency between studies, making it less useful for research purposes. Furthermore, emphasis was placed on making the results relevant to as many clinicians and patients as possible, by not specifying any one treatment protocol or prosthesis in the inclusion and exclusion criteria. However, not specifying a management protocol or prosthesis has resulted in high levels of heterogeneity, making analysis difficult and uncertain. It should also be noted that values calculated are not specific to any one patient, but rather reflect an average rate within the RA population.

Conclusions drawn from temporal analysis are limited by the low number of studies published in the 1980s and 1990s. Similarly, there were a limited number of studies published outside of Europe and North America, which resulted in a risk of bias.

\section{CONCLUSION}

Across four decades, the incidence of THA in patients with RA is 7/1000 PY; and the overall rate of complication rate was $12.8 \%$. Both incidence and overall complications rates of THA in patients with RA have been falling especially in the 2010-2019 decade. However, rates for dislocation increased while infection and aseptic loosening rates remained constant. The results indicate that improvements in medical care have reduced the rate of THA in patients with RA over the last decades, while improvement in surgical and medical management have reduced some, but not all postoperative complication rates for patients with RA.

\section{ACKNOWLEDGEMENTS}

We like to acknowledge Senior Librarian Karen Jones for help within the scope of UWA library support services, specifically in reviewing the search strategy.

Funding. Owen Taylor-Williams was supported by the Cottesloe Women's Service Guild Seaman Scholarly Plus Award in Arthritis Research at the University of Western Australia. No Rapid Service Fee was received by the journal for the publication of this article.

Authorship. All named authors meet the International Committee of Medical Journal Editors (ICMJE) criteria for authorship, take responsibility for the integrity of the work as a whole, and have given their approval for this version to be published.

Disclosures. Owen Taylor-Williams and Charles A. Inderjeeth have nothing to disclose. Johannes Nossent is a member of the journal's Editorial Board.

Compliance with Ethics Guidelines. This article is based on previously conducted studies and does not contain any studies with human participants or animals performed by any of the authors. 
Data Availability. All data generated or analysed is available in this article or the supplementary file.

Open Access. This article is licensed under a Creative Commons Attribution-NonCommercial 4.0 International License, which permits any non-commercial use, sharing, adaptation, distribution and reproduction in any medium or format, as long as you give appropriate credit to the original author(s) and the source, provide a link to the Creative Commons licence, and indicate if changes were made. The images or other third party material in this article are included in the article's Creative Commons licence, unless indicated otherwise in a credit line to the material. If material is not included in the article's Creative Commons licence and your intended use is not permitted by statutory regulation or exceeds the permitted use, you will need to obtain permission directly from the copyright holder. To view a copy of this licence, visit http:// creativecommons.org/licenses/by-nc/4.0/.

\section{REFERENCES}

1. Nikiphorou E, Konan S, MacGregor AJ, Haddad FS, Young A. The surgical treatment of rheumatoid arthritis. Bone Joint J. 2014;96-B(10):1287-9.

2. Raza K, Filer A. The therapeutic window of opportunity in rheumatoid arthritis: does it ever close? Ann Rheum Dis. 2015;74(5):793-4.

3. Singh JA, Saag KG, Bridges SL Jr, et al. 2015 American College of Rheumatology guideline for the treatment of rheumatoid arthritis. Arthritis Rheumatol. 2016;68(1):1-26.

4. Mancuso CA, Ranawat CS, Esdaile JM, Johanson NA, Charlson ME. Indications for total hip and total knee arthroplasties: results of orthopaedic surveys. J Arthroplast. 1996;11(1):34-46.

5. Nikiphorou E, Norton S, Young A, et al. Association between rheumatoid arthritis disease activity, progression of functional limitation and long-term risk of orthopaedic surgery: combined analysis of two prospective cohorts supports EULAR treat to target DAS thresholds. Ann Rheum Dis. 2016;75(12): 2080-6.
6. Young AMD. What have we learnt from early rheumatoid arthritis cohorts? Best Pract Res Clin Rheumatol. 2009;23(1):3-12.

7. Munn Z, Moola S, Lisy K, Riitano D, Tufanaru C. Joanna Briggs Institute Reviewer's Manual: Joana Briggs Institute. 2017. https://reviewersmanual. joannabriggs.org/. Accessed 16 Nov 2019.

8. Singh JA, Vessely MB, Harmsen WS, et al. A population-based study of trends in the use of total hip and total knee arthroplasty, 1969-2008. Mayo Clin Proc. 2010;85(10):898-904.

9. Da Silva E, Doran MF, Crowson CS, O'Fallon WM, Matteson EL. Declining use of orthopedic surgery in patients with rheumatoid arthritis? Results of a long-term, population-based assessment. Arthritis Care Res. 2003;49(2):216-20.

10. Fevang BTS, Lie SA, Havelin LI, Engesaeter LB, Furnes $O$. Reduction in orthopedic surgery among patients with chronic inflammatory joint disease in Norway, 1994-2004. Arthritis Care Res. 2007;57(3): 529-32.

11. Cordtz RL, Hawley S, Prieto-Alhambra D, et al. Incidence of hip and knee replacement in patients with rheumatoid arthritis following the introduction of biological DMARDs: an interrupted timeseries analysis using nationwide Danish healthcare registers. Ann Rheum Dis. 2018;77(5):684-9.

12. Learmonth ID, Young C, Rorabeck C. The operation of the century: total hip replacement. Lancet. 2007;370(9597):1508-19.

13. Richardson SS, Kahlenberg CA, Goodman SM, et al. Inflammatory arthritis is a risk factor for multiple complications after total hip arthroplasty: a population-based comparative study of 68,348 patients. J Arthroplast. 2019;34(6):1150-4.e2.

14. Goodman SM, Figgie MA. Arthroplasty in patients with established rheumatoid arthritis (RA): mitigating risks and optimizing outcomes. Best Pract Res Clin Rheumatol. 2015;29(4):628-42.

15. Michaud K, Fehringer EV, Garvin K, O'Dell JR, Mikuls TR. Rheumatoid arthritis patients are not at increased risk for 30-day cardiovascular events, infections, or mortality after total joint arthroplasty. Arthritis Res Ther. 2013;15(6):R195.

16. Ravi B, Croxford R, Hollands S, et al. Increased risk of complications following total joint arthroplasty in patients with rheumatoid arthritis. Arthritis Rheumatol. 2014;66(2):254-63.

17. Jauregui JJ, Kapadia BH, Dixit A, et al. Thirty-day complications in rheumatoid patients following 
total knee arthroplasty. Clin Rheumatol. 2016;35(3):595-600.

18. Rud-Sørensen C, Pedersen AB, Johnsen SP, Riis AH, Overgaard S. Survival of primary total hip arthroplasty in rheumatoid arthritis patients. Acta Orthop. 2010;81(1):60-5.

19. Ravi B, Escott B, Shah PS, et al. A systematic review and meta-analysis comparing complications following total joint arthroplasty for rheumatoid arthritis versus for osteoarthritis. Arthritis Rheum. 2012;64(12):3839-49.

20. Munn Z, Moola S, Riitano D, Lisy K. The development of a critical appraisal tool for use in systematic reviews addressing questions of prevalence. Int $\mathrm{J}$ Health Policy Manag. 2014;3(3):123-8.

21. Liberati A, Altman DG, Tetzlaff J, et al. The PRISMA statement for reporting systematic reviews and meta-analyses of studies that evaluate healthcare interventions: explanation and elaboration. BMJ. 2009;339:b2700.

22. Hoy D, Brooks P, Woolf A, et al. Assessing risk of bias in prevalence studies: modification of an existing tool and evidence of interrater agreement. J Clin Epidemiol. 2012;65(9):934-9.

23. Davey J, Turner RM, Clarke MJ, Higgins JPT. Characteristics of meta-analyses and their component studies in the Cochrane Database of Systematic Reviews: a cross-sectional, descriptive analysis. BMC Med Res Methodol. 2011;11:160.

24. Aaltonen KJ, Virkki LM, Jämsen E, et al. Do biologic drugs affect the need for and outcome of joint replacements in patients with rheumatoid arthritis? A register-based study. Semin Arthritis Rheum. 2013;43(1):55-62.

25. Eberhardt K, Fex E, Johnsson K, Geborek P. Hip involvement in early rheumatoid arthritis. Ann Rheum Dis. 1995;54(1):45-8.

26. Hawley S, Cordtz R, Dreyer L, et al. Association between NICE guidance on biologic therapies with rates of hip and knee replacement among rheumatoid arthritis patients in England and Wales: an interrupted time-series analysis. Semin Arthritis Rheum. 2018;47(5):605-10.

27. Hawley S, Ali MS, Cordtz R, et al. Impact of TNF inhibitor therapy on joint replacement rates in rheumatoid arthritis: a matched cohort analysis of BSRBR-RA UK registry data. Rheumatology. 2019;58(7):1168-75.

28. Hawley S, Edwards CJ, Arden NK, et al. Descriptive epidemiology of hip and knee replacement in rheumatoid arthritis: an analysis of UK electronic medical records. Semin Arthritis Rheum. 2020;50(2):237-44.

29. Hekmat K, Jacobsson L, Nilsson JA, et al. Decrease in the incidence of total hip arthroplasties in patients with rheumatoid arthritis-results from a well defined population in south Sweden. Arthritis Res Ther. 2010;13(2):R67.

30. James D, Young A, Kulinskaya E, et al. Orthopaedic intervention in early rheumatoid arthritis. Occurrence and predictive factors in an inception cohort of 1064 patients followed for 5 years. Rheumatology. 2004;43(3):369-76.

31. Kapetanovic MC, Lindqvist E, Saxne T, Eberhardt K. Orthopaedic surgery in patients with rheumatoid arthritis over 20 years: prevalence and predictive factors of large joint replacement. Ann Rheum Dis. 2008;67(10):1412-6.

32. Matsumoto $\mathrm{T}$, Nishino J, Izawa $\mathrm{N}$, et al. Trends in treatment, outcomes, and incidence of orthopedic surgery in patients with rheumatoid arthritis: an observational cohort study using the Japanese national database of rheumatic diseases. J Rheumatol. $2017 ; 44(11): 1575-82$.

33. Pantos PG, Tzioufas AG, Panagiotakos DB, Soucacos PN, Moutsopoulos HM. Demographics, clinical characteristics and predictive factors for total knee or hip replacement in patients with rheumatoid arthritis in Greece. Clin Exp Rheumatol. 2013;31(2): 195-200.

34. Shourt CA, Crowson CS, Gabriel SE, Matteson EL. Orthopedic surgery among patients with rheumatoid arthritis 1980-2007: a population-based study focused on surgery rates, sex, and mortality. J Rheumatol. 2012;39(3):481-5.

35. Creighton MG, Callaghan JJ, Olejniczak JP, Johnston RC. Total hip arthroplasty with cement in patients who have rheumatoid arthritis. A minimum ten-year follow-up study. J Bone Joint Surg Am. 1998;80(10):1439-46.

36. Eskelinen A, Paavolainen P, Helenius I, Pulkkinen P, Remes V. Total hip arthroplasty for rheumatoid arthritis in younger patients: 2557 replacements in the Finnish Arthroplasty Register followed for 0-24 years. Acta Orthop. 2006;77(6):853-65.

37. Momohara S, Kawakami K, Iwamoto T, et al. Prosthetic joint infection after total hip or knee arthroplasty in rheumatoid arthritis patients treated with nonbiologic and biologic disease-modifying antirheumatic drugs. Mod Rheumatol. 2011;21(5): 469-75.

38. Furnes O, Lie SA, Espehaug B, et al. Hip disease and the prognosis of total hip replacements. A review of 
53,698 primary total hip replacements reported to the Norwegian Arthroplasty Register 1987-99. J Bone Joint Surg Br. 2001;83(4):579-86.

39. Haraguchi A, Nakashima $Y$, Miyahara $H$, et al. Minimum 10-year results of cementless total hip arthroplasty in patients with rheumatoid arthritis. Mod Rheumatol. 2017;27(4):598-604.

40. Kadota Y, Nishida K, Hashizume K, et al. Risk factors for surgical site infection and delayed wound healing after orthopedic surgery in rheumatoid arthritis patients. Mod Rheumatol. 2016;26(1):68-74.

41. Katsimihas M, Taylor AH, Lee MB, Sarangi PP, Learmonth ID. Cementless acetabular replacement in patients with rheumatoid arthritis: a 6- to 14-year prospective study. J Arthroplasty. 2003;18(1):16-22.

42. Kesteris U, Robertsson O, Wingstrand H, Onnerfalt R. Cumulative revision rate with the Scan Hip Classic I total hip prosthesis. 1660 cases followed for 2-12 years. Acta Orthop Scandinavica. 1998;69(2): $133-7$.

43. Learmonth ID, Sarangi PP, Lee MB, Smith EJ. Cementless replacement of the acetabulum at total hip arthroplasty in rheumatoid arthritis. J Orthop Rheumatol. 1996;9(1):33-6.

44. Lehtimaki MY, Kautiainen H, Lehto MUK, Hamalainen MMJ. Charnley low-friction arthroplasty in rheumatoid patients: a survival study up to 20 years. J Arthroplasty. 1999;14(6):657-61.

45. Parvizi J, Johnson BG, Rowland C, Ereth MH, Lewallen DG. Thirty-day mortality after elective total hip arthroplasty. J Bone Joint Surg Am. 2001;83(10):1524-8.

46. Rosenberg WW, Schreurs BW, de Waal Malefijt MC, Veth RP, Slooff TJ. Impacted morsellized bone grafting and cemented primary total hip arthroplasty for acetabular protrusion in patients with rheumatoid arthritis: an 8- to 18-year follow-up study of 36 hips. Acta Orthop Scand. 2000;71(2): $143-6$.

47. Schnaser EA, Browne JA, Padgett DE, Figgie MP, D'Apuzzo MR. Perioperative complications in patients with inflammatory arthropathy undergoing total hip arthroplasty. J Arthroplasty. 2016;31(10):2286-90.

48. Stundner O, Chiu Y-L, Sun X, et al. Perioperative outcomes in patients with rheumatoid versus osteoarthritis for total hip arthroplasty: a population-based study. Clin Exp Rheumatol. 2013;31(6): 889-95.

49. van Stralen GMJ, Struben PJ, van Loon CJM. The incidence of dislocation after primary total hip arthroplasty using posterior approach with posterior soft-tissue repair. Arch Orthop Trauma Surg. 2003;123(5):219-22.

50. Zwartele R, Peters A, Brouwers J, et al. Long-term results of cementless primary total hip arthroplasty with a threaded cup and a tapered, rectangular titanium stem in rheumatoid arthritis and osteoarthritis. Int Orthop. 2008;32(5):581-7.

51. Fink B, Siegmuller C, Schneider T, et al. Short- and medium-term results of the thrust plate prosthesis in patients with polyarthritis. Arch Orthop Trauma Surg. 2000;120(5-6):294-8.

52. Niggemeyer O, Steinhagen J, Ruether W. Long-term results of the thrust plate prosthesis in patients with rheumatoid arthritis: a minimum 10-year followup. J Orthop Sci. 2010;15(6):772-80.

53. Onsten I, Besjakov J, Carlsson AS. Improved radiographic survival of the Charnley prosthesis in rheumatoid arthritis and osteoarthritis. Results of new versus old operative techniques in 402 hips. J Arthroplasty. 1994;9(1):3-8.

54. Partio E, von Bonsdorff $\mathrm{H}$, Wirta J, Avikainen V. Survival of the Lubinus hip prosthesis. An eight- to 12-year follow-up evaluation of 444 cases. Clin Orthop Related Res. 1994;303:140-6.

55. Unger AS, Ranawat CS, Johanson NA. Total hip arthroplasty in rheumatoid arthritis: a long-term follow-up study. J Arthroplasty. 1987;2(3):191-7.

56. Hayashi S, Hashimoto S, Takayama K, et al. Risk factors for late deep infection after total hip arthroplasty in patients with rheumatoid arthritis. Acta Rheumatol Portugesa. 2017;42(2):150-4.

57. Lakatos J, Csakanyi L. Comparison of complications of total hip arthroplasty in rheumatoid arthritis, ankylosing spondylitis, and osteoarthritis. Orthopedics. $1991 ; 14(1): 55-7$.

58. Learmonth ID, Dall DM, Solomon M, Heywood AWB. A comparison of the results of Charnley low friction arthroplasty in rheumatoid arthritis and osteoarthritis. J Orthop Rheumatol. 1991;4(1): $47-55$.

59. Makela KT, Eskelinen A, Pulkkinen $\mathrm{P}$, et al. Cemented versus cementless total hip replacements in patients fifty-five years of age or older with rheumatoid arthritis. J Bone Joint Surg Am. 2011;93(2):178-86.

60. White RH, McCurdy SA, Marder RA. Early morbidity after total hip replacement: rheumatoid arthritis versus osteoarthritis. J Gen Intern Med. 1990;5(4): 304-9. 
61. Kirk PG, Rorabeck CH, Bourne RB, Burkart B. Total hip arthroplasty in rheumatoid arthritis: comparison of cemented and uncemented implants. Can J Surg. 1993;36(3):229-32.

62. Poss R, Maloney JP, Ewald FC, et al. Six- to 11-year results of total hip arthroplasty in rheumatoid arthritis. Clin Orthop Relat Res. 1984;182:109-16.

63. Effenberger H, Ramsauer T, Böhm G, et al. Successful hip arthroplasty using cementless titanium implants in rheumatoid arthritis. Arch Orthop Trauma Surg. 2002;122(2):80-7.

64. Burn E, Edwards CJ, Murray DW, et al. The impact of rheumatoid arthritis on the risk of adverse events following joint replacement: a real-world cohort study. Clin Epidemiol. 2018;10:697-704.

65. Massardo L, Gabriel SE, Crowson CS, O'Fallon WM, Matteson EL. A population based assessment of the use of orthopedic surgery in patients with rheumatoid arthritis. J Rheumatol. 2002;29(1):52-6.

66. Crawford RW, Murray DW. Total hip replacement: indications for surgery and risk factors for failure. Ann Rheum Dis. 1997;56(8):455-7.

67. Skyttä ET, Honkanen PB, Eskelinen A, Huhtala H, Remes V. Fewer and older patients with rheumatoid arthritis need total knee replacement. Scand J Rheumatol. 2012;41(5):345-9.

68. Jämsen E, Virta LJ, Hakala M, et al. The decline in joint replacement surgery in rheumatoid arthritis is associated with a concomitant increase in the intensity of anti-rheumatic therapy: a nationwide register-based study from 1995 through 2010. Acta Orthop. 2013;84(4):331-7.

69. Young BL, Watson SL, Perez JL, et al. Trends in joint replacement surgery in patients with rheumatoid arthritis. J Rheumatol. 2018;45(2):158-64.

70. Nikiphorou E, Carpenter L, Morris S, et al. Hand and foot surgery rates in rheumatoid arthritis have declined from 1986 to 2011, but large-joint replacement rates remain unchanged: results from two UK inception cohorts. Arthritis Rheumatol. 2014;66(5):1081-9.

71. American College of Rheumatology Subcommittee on Rheumatoid Arthritis Guidelines. Guidelines for the management of rheumatoid arthritis: 2002 Update. Arthritis Rheum. 2002;46(2):328-46.

72. Curtis JR, Singh JA. Use of biologics in rheumatoid arthritis: current and emerging paradigms of care. Clin Ther. 2011;33(6):679-707.
73. Weinblatt ME. Methotrexate in rheumatoid arthritis: a quarter century of development. Trans Am Clin Climatol Assoc. 2013;124:16-25.

74. Steffen A, Holstiege J, Klimke K, Akmatov MK, Bätzing J. Patterns of the initiation of diseasemodifying antirheumatic drugs in incident rheumatoid arthritis: a German perspective based on nationwide ambulatory drug prescription data. Rheumatol Int. 2018;38(11):2111-200.

75. Espinoza F, Fabre S, Pers Y-M. Remission-induction therapies for early rheumatoid arthritis: evidence to date and clinical implications. Ther Adv Musculoskel Dis. 2016;8(4):107-18.

76. Kimsey L, Weissman JS, Patel A, et al. Delay in initiation of DMARD or anti-inflammatory therapy in patients newly diagnosed with rheumatoid arthritis: an analysis of United States Military Health System TRICARE beneficiaries. Semin Arthritis Rheum. 2019;48(5):821-7.

77. Saag KG, Teng GG, Patkar NM, et al. American College of Rheumatology 2008 recommendations for the use of nonbiologic and biologic diseasemodifying antirheumatic drugs in rheumatoid arthritis. Arthritis Care Res. 2008;59(6):762-84.

78. Harrold LR, Peterson D, Beard AJ, Gurwitz JH, Briesacher BA. Time trends in medication use and expenditures in older patients with rheumatoid arthritis. Am J Med. 2012;125(9):937.e9-.e15.

79. Palm TM, Kaarela K, Hakala MS, et al. Need and sequence of large joint replacements in rheumatoid arthritis. A 25-year follow-up study. Clin Exp Rheumatol. 2002;20(3):392-4.

80. Minichiello E, Semerano L, Boissier MC. Time trends in the incidence, prevalence, and severity of rheumatoid arthritis: a systematic literature review. Joint Bone Spine. 2016;83(6):625-30.

81. Diffin JG, Lunt M, Marshall T, et al. Has the severity of rheumatoid arthritis at presentation diminished over time? J Rheumatol. 2014;41(8):1590-9.

82. Alamanos Y, Voulgari PV, Drosos AA (2006) Incidence and prevalence of rheumatoid arthritis, based on the 1987 American college of rheumatology criteria: a systematic review. Semin Arthr Rheum 36(3):182-8.

83. Tobón GJ, Youinou P, Saraux A (2010) The environment, geo-epidemiology, and autoimmune disease: rheumatoid arthritis. J Autoimmun 35(1): 10-14.

84. Merx H, Dreinhöfer K, Schräder P, et al. International variation in hip replacement rates. Ann Rheum Dis. 2003;62(3):222-6. 
85. Wetterholm M, Turkiewicz A, Stigmar K, Hubertsson J, Englund M. The rate of joint replacement in osteoarthritis depends on the patient's socioeconomic status. Acta Orthop. 2016;87(3):245-51.

86. Judge A, Welton NJ, Sandhu J, Ben-Shlomo Y. Equity in access to total joint replacement of the hip and knee in England: cross sectional study. BMJ. 2010;341:c4092.

87. Cushner F, Agnelli G, FitzGerald G, Warwick D. Complications and functional outcomes after total hip arthroplasty and total knee arthroplasty: results from the Global Orthopaedic Registry (GLORY). Am J Orthopaedics. 2010;39(9):22-8.

88. Katz JN, Losina E, Barrett J, et al. Association between hospital and surgeon procedure volume and outcomes of total hip replacement in the United States Medicare population. J Bone Joint Surg Am. 2001;83(11):1622-9.

89. Yasunaga $H$, Tsuchiya $K$, Matsuyama $Y$, Ohe $K$. High-volume surgeons in regard to reductions in operating time, blood loss, and postoperative complications for total hip arthroplasty. J Orthop Sci. 2009;14(1):3-9.

90. Dolan AL, Moniz C, Abraha H, Pitt P. Does active treatment of rheumatoid arthritis limit disease-associated bone loss? Rheumatology. 2002;41(9): 1047-51.

91. Ozen G, Pedro S, Wolfe F, Michaud K. Medications associated with fracture risk in patients with rheumatoid arthritis. Ann Rheum Dis. 2019;78(8): 1041-7.

92. Bohler C, Weimann P, Alasti F, et al. Rheumatoid arthritis disease activity and the risk of aseptic arthroplasty loosening. Semin Arthritis Rheum. 2020;50(2):245-51.

93. Raterman HG, Lems WF. Pharmacological management of osteoporosis in rheumatoid arthritis patients: a review of the literature and practical guide. Drugs Aging. 2019;36(12):1061-72.

94. Meek R, Norwood T, Smith R, Brenkel IJ, Howie CR. The risk of peri-prosthetic fracture after primary and revision total hip and knee replacement. J Bone Joint Surg Br. 2011;93(1):96-101.

95. Bourne RB, Maloney WJ, Wright JG. An AOA critical issue. The outcome of the outcomes movement. J Bone Joint Surg Am. 2004;86(3):633-40.

96. Bozic KJ, Kurtz SM, Lau E, et al. The epidemiology of revision total hip arthroplasty in the United States. J Bone Joint Surg Am. 2009;91(1):128-33.
97. Rowan FE, Benjamin B, Pietrak JR, Haddad FS. Prevention of dislocation after total hip arthroplasty. J Arthroplasty. 2018;33(5):1316-24.

98. Petis S, Howard JL, Lanting BL, Vasarhelyi EM. Surgical approach in primary total hip arthroplasty: anatomy, technique and clinical outcomes. Can J Surg. 2015;58(2):128-39.

99. Tsikandylakis G, Mohaddes M, Cnudde P, et al. Head size in primary total hip arthroplasty. EFORT Open Rev. 2018;3(5):225-31.

100. Crowninshield RD, Rosenberg AG, Sporer SM. Changing demographics of patients with total joint replacement. Clin Orthop Relat Res. 2006;443: 266-72.

101. Fevang BTS, Lie SA, Havelin LI, Engesaeter LB, Furnes O. Improved results of primary total hip replacement: results from the Norwegian Arthroplasty Register, 1987-2007. Acta Orthop. 2010;81(6):649-59.

102. Karachalios T, Komnos G, Koutalos A. Total hip arthroplasty: survival and modes of failure. EFORT Open Rev. 2018;3(5):232-9.

103. Biau DJ, Leclerc P, Marmor S, et al. Monitoring the one year postoperative infection rate after primary total hip replacement. Int Orthop. 2012;36(6): 1155-61.

104. Somayaji R, Barnabe C, Martin L. Risk factors for infection following total joint arthroplasty in rheumatoid arthritis. Open Rheumatol J. 2013;7: $119-24$.

105. Lacaille D, Guh DP, Abrahamowicz M, Anis AH, Esdaile JM. Use of nonbiologic disease-modifying antirheumatic drugs and risk of infection in patients with rheumatoid arthritis. Arthritis Care Res. 2008;59(8):1074-81.

106. Makol A, Davis JM 3rd, Crowson CS, et al. Time trends in glucocorticoid use in rheumatoid arthritis: results from a population-based inception cohort, 1980-1994 versus 1995-2007. Arthritis Care Res. 2014;66(10):1482-8.

107. Ozen G, Pedro S, England BR, et al. Risk of serious infection in patients with rheumatoid arthritis treated with biologic versus nonbiologic diseasemodifying antirheumatic drugs. ACR Open Rheumatol. 2019;1(7):424-32.

108. Ramiro S, Sepriano A, Chatzidionysiou K, et al. Safety of synthetic and biological DMARDs: a systematic literature review informing the 2016 update of the EULAR recommendations for management of rheumatoid arthritis. Ann Rheum Dis. 2017;76(6): 1101-36. 
109. Ito H, Kojima M, Nishida K, et al. Postoperative complications in patients with rheumatoid arthritis using a biological agent-a systematic review and meta-analysis. Mod Rheumatol. 2015;25(5):672-8.

110. Goodman SM, Menon I, Christos PJ, Smethurst R, Bykerk VP. Management of perioperative tumour necrosis factor $\alpha$ inhibitors in rheumatoid arthritis patients undergoing arthroplasty: a systematic review and meta-analysis. Rheumatology. 2015;55(3):573-82.

111. George MD, Baker JF, Winthrop K, et al. Risk of biologics and glucocorticoids in patients with rheumatoid arthritis undergoing arthroplasty: a cohort study. Ann Intern Med. 2019;170(12): 825-36.

112. Lindahl H. Epidemiology of periprosthetic femur fracture around a total hip arthroplasty. Injury. 2007;38(6):651-4.

113. Falck-Ytter Y, Francis CW, Johanson NA, et al. Prevention of VTE in orthopedic surgery patients: antithrombotic therapy and prevention of thrombosis, 9th ed: American College of Chest Physicians evidence-based clinical practice guidelines. Chest. 2012;141(2 Suppl):e278S-e325S.

114. Xing KH, Morrison G, Lim W, et al. Has the incidence of deep vein thrombosis in patients undergoing total hip/knee arthroplasty changed over time? A systematic review of randomized controlled trials. Thromb Res. 2008;123(1):24-34.

115. Flevas DA, Megaloikonomos PD, Dimopoulos L, et al. Thromboembolism prophylaxis in orthopaedics: an update. EFORT Open Rev. 2018;3(4): 136-48.

116. Farfan M, Bautista M, Bonilla G, et al. Worldwide adherence to ACCP guidelines for thromboprophylaxis after major orthopedic surgery: a systematic review of the literature and meta-analysis. Thromb Res. 2016;141:163-70.

117. Partridge T, Jameson S, Baker P, et al. Ten-year trends in medical complications following 540,623 primary total hip replacements from a national database. J Bone Joint Surg Am. 2018;100(5):360-7.

118. Xu K, Chan NC, Ibrahim Q, et al. Reduction in mortality following elective major hip and knee surgery: a systematic review and meta-analysis. Thromb Haemost. 2019;119(4):668-74.

119. Stundner O, Danninger T, Chiu Y-L, et al. Rheumatoid arthritis vs osteoarthritis in patients receiving total knee arthroplasty: perioperative outcomes. J Arthroplasty. 2014;29(2):308-13. 